Relations industrielles

Industrial Relations

\title{
Aperçu sur la conciliation
}

\section{Louis-de-Gonzague Gosselin}

Volume 9, numéro 1, décembre 1953

URI : https://id.erudit.org/iderudit/1022916ar

DOI : https://doi.org/10.7202/1022916ar

Aller au sommaire du numéro

\section{Éditeur(s)}

Département des relations industrielles de l’Université Laval

ISSN

0034-379X (imprimé)

1703-8138 (numérique)

Découvrir la revue

Citer ce document

Gosselin, L.-d.-G. (1953). Aperçu sur la conciliation. Relations industrielles /

Industrial Relations, 9(1), 69-76. https://doi.org/10.7202/1022916ar

Tous droits réservés @ Département des relations industrielles de l’Université Laval, 1953
Ce document est protégé par la loi sur le droit d'auteur. L'utilisation des services d'Érudit (y compris la reproduction) est assujettie à sa politique d'utilisation que vous pouvez consulter en ligne.

https://apropos.erudit.org/fr/usagers/politique-dutilisation/ 


\section{INFORMATIONS}

\section{Aperçu sur la conciliation}

Le Service de conciliation et d'arbitrage dans le domaine du travail participe à la mise à exécution, dans la province, de la Loi des Différends Ouvriers, de la Loi des Relations Ouvrières et de la Loi des différends entre les services publics et leurs salariés, tout en collaborant avec la Commission de relations ouvrières du Québec.

Avant de démontrer le travail proprement dit de ce Service, il me semble opportun de spécifier que la procédure qui se situe entre les négociations directes et l'arbitrage s'appelle la conciliation et peut avoir lieu lors des négociations en vue d'une première convention collective ou lors d'un renouvellement, lors de la discussion d'un grief survenu au cours de la durée d'une convention, lors de l'étude d'une clause ouverte ou lors des pourparlers après un arbitrage.

Il suffit d'en consulter les rapports, soit depuis le ler avril 1945 au 31 mars 1953, pour cons ater que les interventions conciliatrices du Service prennent l'allure d'une courbe ascendante, puisque pour l'année 1945, elles se chiffrent à 138, couvrant un total de 266 employeurs et de 22,355 employés, alors que pour l année fiscale se terminant le 31 mars 1953 , elles se chiffrent à 751 , couvrant un total de 14,598 établissements et de 266,300 employés. Il est à no er que depuis le ler avril 1945 jusqu'au 31 mars 1953, ce Service a effectué un total de 3,234 interventions conciliatrices, couvrant 43,925 employeurs et $1,040,321$ employés.

Si les interventions conciliatrices ne cessent d'augmenter (voir tableaux 1a, 1b, 1c), nous ne pouvons pas conclure que les relations patronales-ouvrières vont de plus en plus mal; mais cette nécessité de plus en plus remarquable de l'intervention conciliatrice est-elle une indication, chez nous, de l'amélioration générale des relations entre employeurs et employés, de la mise à la page de la législation qui les régit, d'une meilleure compréhension du problème social de la part du patronat et des chefs de la classe ouvrière?

Il est bien évident que l'organisation ouvrière a pris une grande expansion. D'ailleurs, les différentes campagnes d'organisation lancées par les différents mouvements ouvriers sont là pour le prouver. Nous sommes devant un fait indiscutable mais bien réel: il y a plus d'employés organisés et plus de syndicats reconnus en 1953 que durant les années précédentes, puisque en 1943-44, il y eut 44 conciliations proprement dites qui furent faites en vue de règlement de conflits. Il est iritéressant de noter ici que dans le passé, soit de 1931 à 1943, les interventions du gouvernement en matière de conciliation étaient faites en vue de régler des conflits qui étaient sur le point d'éclater ou qui avaient éclaté. Ainsi, en 1941-42, à la suite d'arrêts de travail, 56 interventions étaient faites par le gouvernement provincial, et durant l'année 1942-43, 64 interventions furent effectuées.

Le pourcentage des interventions jugées statisfaisantes par les parties pourrait représenter une courbe irrégulière variant entre 42 et $64.14 \%$. L'an denier, les interventions conciliatrices réglées à la satisfaction des parties s'élevaient à $57.5 \%$, alors que cette année, les interventions conciliatrices réglées à la satisfaction des parties s'élèvent à $64.14 \%$, ce qui marque le plus haut pourcentage atteint.

D'après des compilations faites sur les interventions conciliatrices groupées selon les principaux sujets en litige (voir Tableau 2), il ressort que dans $\mathbf{6 6 . 4 4 \%}$ des cas soumis, la question des salaires a été discutée, alors que $18.64 \%$ ont été soumis pour l'étude de toute la convention collective. Dans $17 \%$ des cas, la question des congés payés a été discutée, alors que la question des vacances payées l'a été dans $22.1 \%$ des cas soumis. L'item «Sécurité Syndicale 》 a été discuté dans 


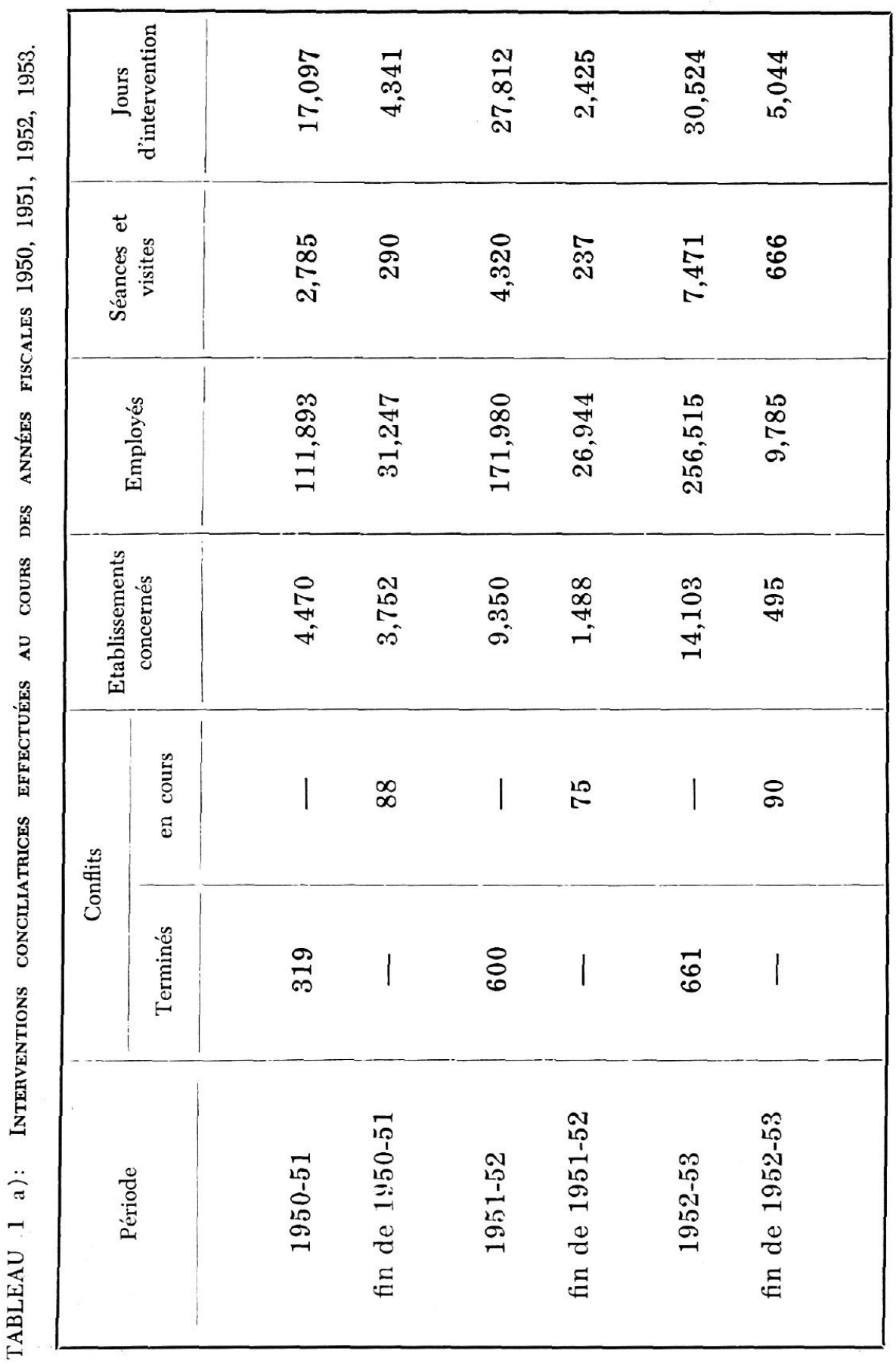




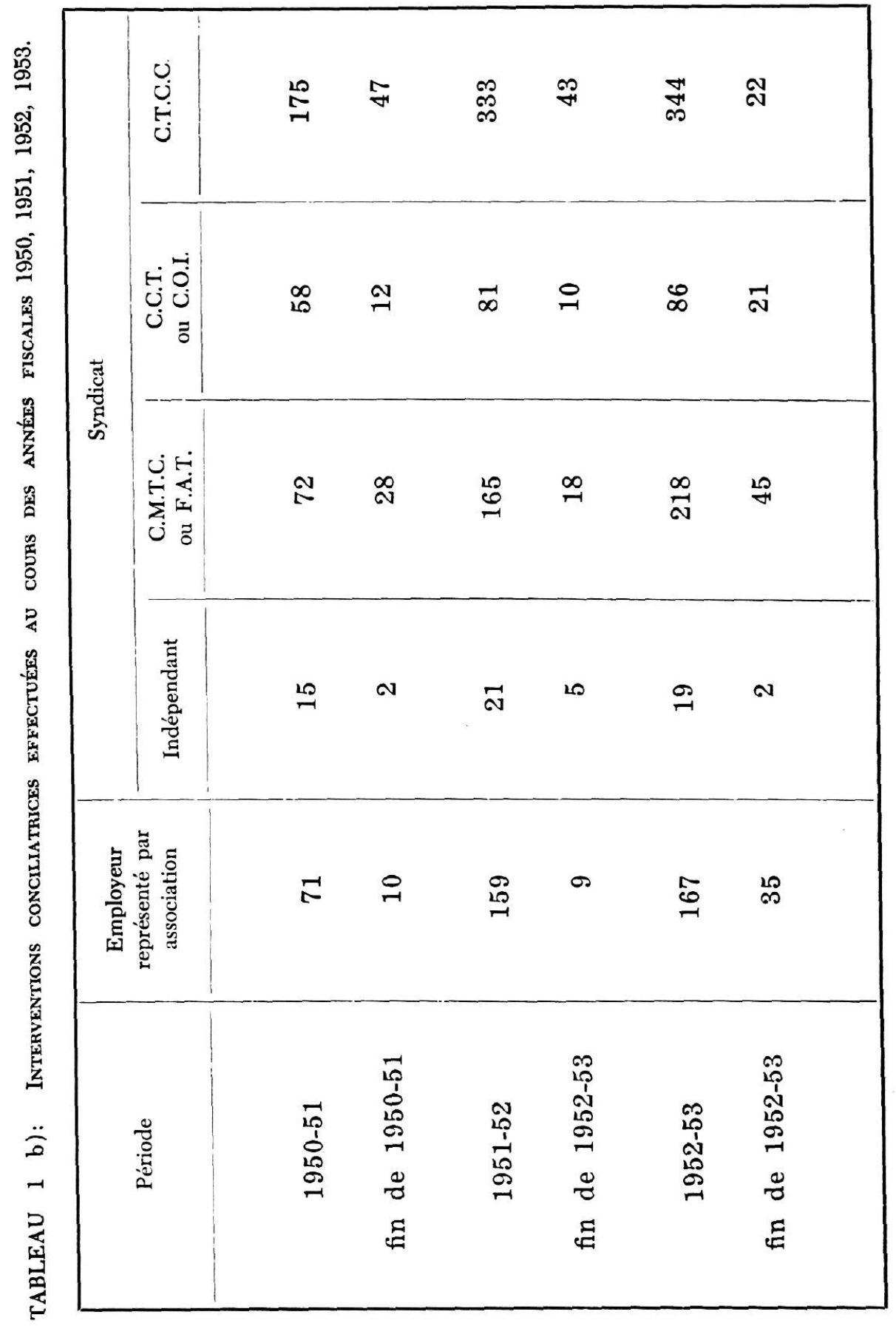




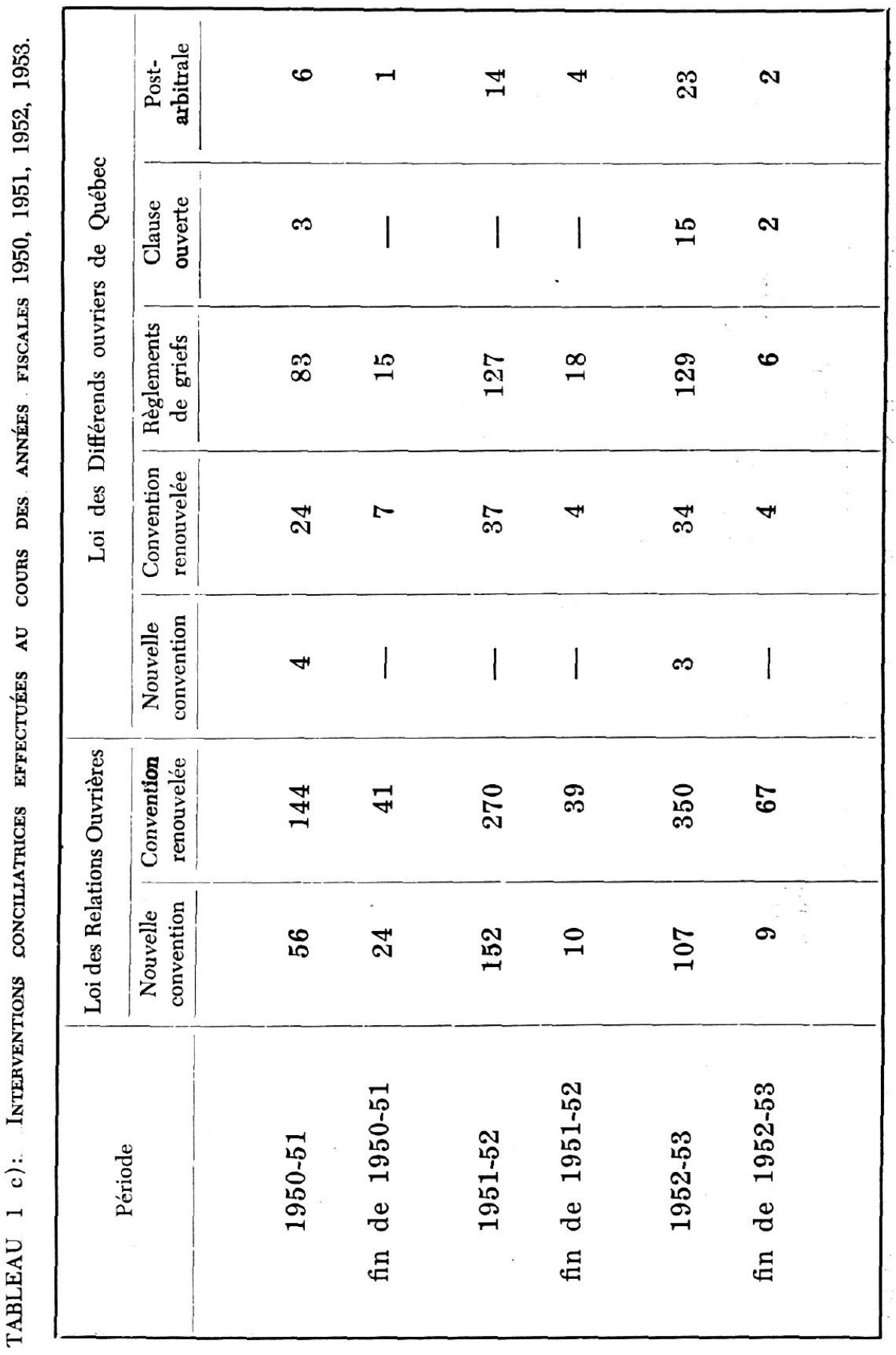




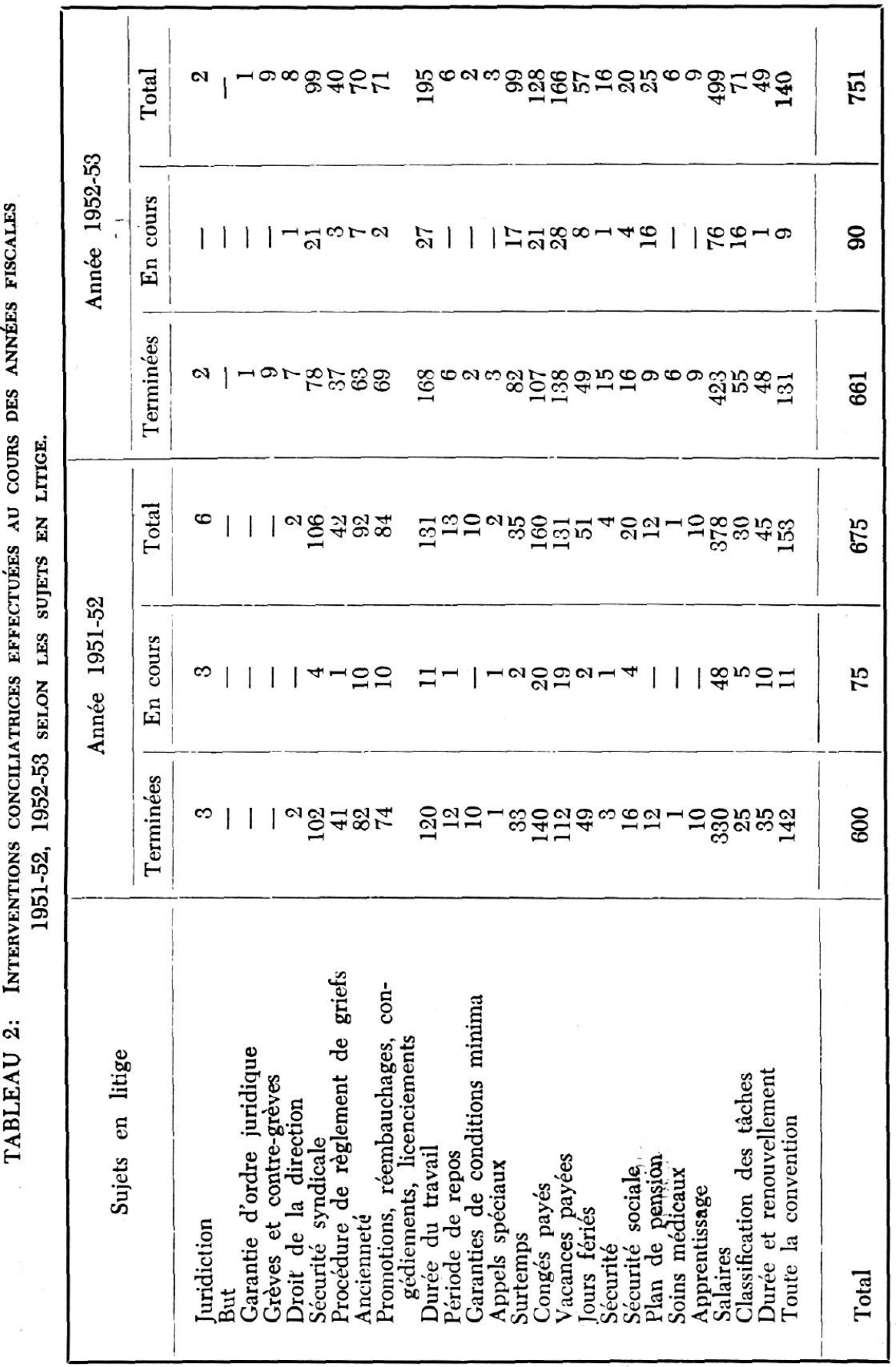




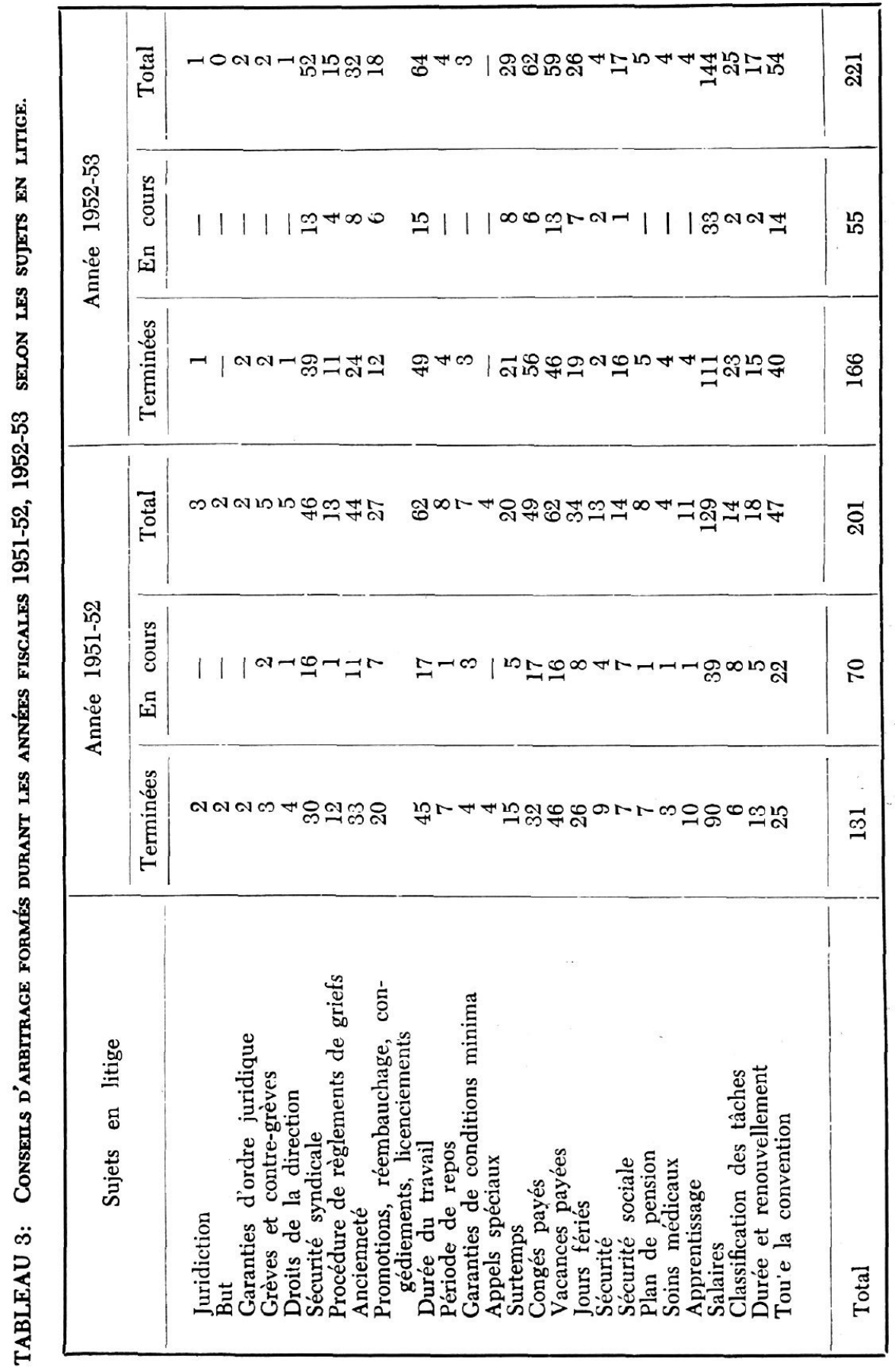


$13.8 \%$ des cas soumis. Il s'ensuit que les salaires, la durée de travail, les vacances payées, l'étude de toute la convention, les congés payés, la sécurité syndicale, le surtemps, les promotions, congédiements et licenciements, et l'ancienneté sont les points litigieux les plus souvent traités.

Le travail du Service ne s'est pas limité aux interventions conciliatrices, bien que ce soit la partie la plus impor ante de son champ d'action. Il me semble donc à propos de signaler qu'au cours de la dernière année fiscale, soit du ler avril 1952 au 31 mars 1953, il fut créé 221 conseils d'arbitrage, englobant 2,153 employeurs et 82,786 employés. Au cours des années précédentes, le nombre des conseils d'arbitrage a varié entre 54 et 201 , soit de 1945 à 1952 , couvrant de 255 à 2,473 employeurs et de 14,549 à 89,624 employés. Même si le nombre des conseils d'arbitrage a augmenté durant l'année fiscale 1952-53, il n'en reste pas moins que seulement $26.32 \%$ des interventions conciliatrices ont dû aboutir à l'arbitrage. De ce fait, le nombre des conseils d'arbitrage résultant des interventions conciliatrices a l'allure d'une courbe irrégulière variant de 23 à $36 \%$. Le Tableau no 4 , ci-joint, donnera une idée du nombre des conseils d'arbitrage formés de 1941 à 1951, année du calendrier.

TABLEAU 4: Nombre de conseils d'arbitrage formés de 1941 à 1951.

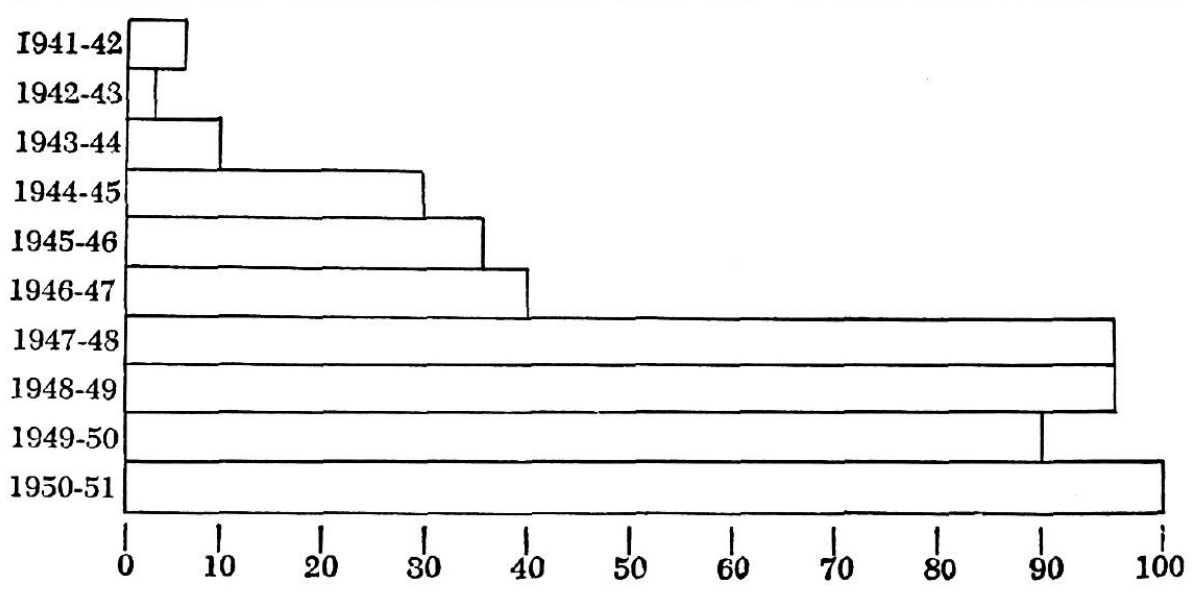

Si nous analysons les sujets litigieux soumis à l'étude des conseils d'arbitrage durant la dernière année fiscale, nous constatons que la question des salaires a été discutée dans $65.15 \%$ des cas; que toute la convention collective a été soumise à l'étude dans $24.43 \%$ des cas. Il ressort également que les sujets litigieux les plus régulièrement soumis à l'étude des conseils sont: les salaires $(65.15 \%)$, la durée du travail $(28.96 \%)$, les congés payés $(28.05 \%)$, les vacances payées $(26.69 \%)$ et l'étude de toute la convention ( $24.43 \%$ des cas).

Plusieurs se demanderont comment il se fait que tant de litiges soient soumis à l'arbitrage. Dans les cas portés à l'arbitrage, l'on peut dire que certains sont pratiquement insolubles en soi. Même si de nos jours on apporte beaucoup plus de considération au rôle joué par le conciliateur, il y en a encore qui ont un certain manque de confiance dans la compétence et dans le rôle du conciliateur. On a vu, dans certains cas, que l'arbitrage apportait à peu près les mêmes résultats que les solutions données par le conciliateur même à la phase de la conciliation. Il reste aussi le fait que le conciliateur n'impose pas de décision, mais suggère 
seulement ce qui amène certaines parties à désirer passer par toutes les procédures, ce qui peut amener une certaine erreur psychologique. De façon générale, l'on peut dire que les conseils d'arbitrage n'ont pas augmenté comparativement à l'augmentation des cas de conciliation.

De plus, les conciliateurs doivent s'occuper, de façon officielle ou officieuse, des conflits qui surviennent dans la province. Ainsi, durant l'année fiscale 1952-53, 39 cas de grève ou contre-grève concernant 41 employeurs et couvrant 17,602 employés ont été portés à leur attention. Pour terminer l'énumération globale du travail accompli, des enquêtes sont effectuées par le Service de conciliation pour la Commission de relations ouvrières et des cas de comité paritaire sont portés à son étude, soit pour fins d'enquête, soit pour l'étude de l'état des métiers, soit pour recommandation, ou modifications à apporter à un décret.

Les différents tableaux annexés, et énoncés mettent en lumière le rôle du Service de conciliation et la tâche extrêmement difficile de la participation à la prévention et' au règlement des difficultés du monde ouvrier.

Louis-De-Gonzague Gosselin, statisticien, Service de conciliation et d'arbitrage de la province de Québec.

\section{Relations humaines dans l'entreprise}

Récemment, sa Sainteté le Pape Pie XII prononçait des discours intéressants sur ce sujet en s'adressant aux industriels américains et auıx ingénieurs.

\section{I-Aux industriels américains *}

Il est réconfortant de constater que le problème délicat mais critique des relations humaines dans l'usine, dans la fonderie, et dans le bureau, est en train d'attirer l'attention qu'il mérite dans vos réunions avec vos collègues de l'étranger. Ces fréquents et cordiaux contacts croissants entre les représentants des moyennes et petites entreprises privées, harcelées dans le monde entier par des problèmes communs à l'humanité, spécialement en ce qui concerne la famille, ne peuvent qu'améliorer les systèmes de production et de distribution.

A condition toutefois que vous fassiez passer au premier plan les choses principales, au bureau comme vous le faites à la maison. Il y a quelques années, Notre Prédécesseur d'heureuse mémoire dut exprimer l'amer regret que \& la matière sort ennoblie de l'usine, et les hommes trop souvent avilis 》 (Quadragesimo Anno). Non, avant d'être un moule pour le métal, l'usine comme toute association humaine, est un moule pour les hommes. Pour animer le groupe industriel ou l'unir, il y a l'âme du travailleur, aussi bien employeur qu'employé avec toutes ses espérances et ses craintes humaines, avec son noble destin et ses prérogatives inaliénables. La sécurité et l'efficacité dans une affaire sont le résultat d'un sens de justice et d'un souci de bonne entente entre ceux qui unissent leurs efforts humains - souvent leur courage héroïque - pour réaliser et maintenir ce que vous. Américains, appelez "une affaire qui marche ». Devons-Nous vous rappeler une fois de plus que les bénéfices, les salaires et les bilans de production sont tous en fonction de l'activité humaine - qui comprend les droits et les sentiments humains - et non point le contraire.

Nous espérons que vous aurez été encouragés de trouver vos collègues européens unis à vous dans leur volonté de résister courageusement à ce processus de déper-

(*) L’Osservatore Romano, 16 octobre 1953. 